\title{
Multi-sensor Information Fusion Steady-State Kalman Estimator for Systems with System Errors and Sensor Errors
}

\author{
Yun $\mathrm{Li}^{1,2}$, Ming Zhao ${ }^{1}$, Gang $\mathrm{Hao}^{2} *$ \\ 1 School of Computer and Information Engineering \\ Harbin University of Commerce \\ Heilongjiang, Harbin, 150001, China \\ liyunhd@sina.com \\ 2 Electronic Engineering Institute \\ Heilongjiang University \\ Heilongjiang, Harbin, 150080, China \\ haogang@hlju.edu.cn
}

\begin{abstract}
In this paper, a multi-sensor information fusion steady-state Kalman estimator for discrete time stochastic linear systems with system errors and sensor errors is presented. Gevers-Wouters $(G-W)$ algorithm is used in this paper. Steady-state Kalman estimator is presented in this paper avoids the complex Diophantine equation, and it is based on the ARMA model to compute the steady-state Kalman estimators gain, further the Lyapunov equation is used to estimate the variance matrix and covariance matrix of estimation error. So this algorithm can obviously reduce the computational burden. In order to improve the estimation accuracy, the multi-sensor information fusion method is adopted. The fusion method includes weighted measurement fusion, weighted by scalars and the covariance intersection fusion. Under the linear minimum variance optimal information fusion criterion, the calculation formula of optimal weighting coefficients have be given in order to realize scalars weighted. To avoid the calculation of cross-covariance matrices, another distributed fusion filter is also presented by using the covariance intersection fusion algorithm, which can reduce the computational burden. And the relationship between the accuracy and the computation complexities among the three fusion algorithm are analyzed. A simulation example of the target tracking controllable system with two sensors shows its effectiveness and correctness.
\end{abstract}

Keywords: multi-sensor information fusion; the linear minimum variance; the steadystate estimator; system errors and sensor errors; Gevers-Wouters algorithm

\section{Introduction}

Classic Kalman filtering is a time varying recursive filter. The optimal Kalman filter is required to compute the gain matrix, the variance matrix and covariance matrix of estimation error at every moment based on Riccati equation, which brings a large computational burden. The steady state Kalman estimators based on modern time series analysis method, which is based on the ARMA model to obtain the steady-state Kalman estimators gain. And the Lyapunov equation is used to estimate the variance matrix and the covariance matrix of the estimation error. This method completely avoids the Riccati equation, and uses the the ARMA model to replace the Riccati equation. The method can obviously reduce the computational burden, and the method can be used to design the self-tuning steady-state Kalman estimators of unknown noise statistics system $^{\text {Error! Reference source not found. } \sim \text { Error! Reference source not found. }}$

Gang Hao is the corresponding author. 
In order to improve the estimation accuracy of the sensor, multi-sensor information fusion is presented. Multi-sensor information fusion is how to combine the local state estimators or local measurements which is obtained from each sensor, to obtain the fused state estimator. And its accuracy is higher than that of each local estimator. There are two common types of information fusion method. One is state fusion, the other is measurement fusion. The state fusion method is also divided into centralized Kalman filtering and distributed Kalman filtering. Although the centralized Kalman filter can obtain the global optimal fusion state estimation in theory, and it has the disadvantage of large computation burden and poor fault tolerance, and the distributed Kalman filtering information fusion can overcome these drawbacks. Distributed fusion Kalman filtering under linear minimum variance rules has three information fusion algorithms: the matrix weighted, scalar weighted and diagonal matrix weighted. The distributed fusion estimation needs to calculate the cross-covariances of local estimate $^{\text {Error! Reference source not found. - Error! Reference source not found. }}$. However, in many theoretical and application problems, the cross-covariances are unknown, or it is very difficult to compute the cross-covariances, or can't calculate the cross-covariances. If the crosscovariances are neglected, it will lead the increase of the variance of the local estimator error, even the divergence of the estimator.

In order to overcome the disadvantages and limitations, Jeffrey K. Uhlman proposed the covariance intersection information fusion method. It is also a distributed information fusion method, and it completely avoids the unknown cross-covariance identification and calculation. It can deal with the fusion estimation problem for the system with unknown covariance. This algorithm avoids computing the covariance, so it can reduce the computational burden. Further the algorithm can be used to fusion estimator for nonlinear systems ${ }^{\text {Error! Reference source not found. } \sim \text { Error! Reference source not found. }}$

In this paper, a multi-sensor information fusion steady-state Kalman estimator for discrete time stochastic linear systems with system errors and sensor errors is presented. Firstly, steady-state Kalman estimator is presented in this paper can obviously reduce the computational burden. Secondly, the fusion method includes weighted measurement fusion, weighted by scalars and the covariance intersection fusion. The accuracy of above three kinds of weighted fusion steady-state Kalman estimator from high to low is weighted measurement fusion, scalars weighted and the covariance intersection fusion. But the computational burden is on the contrary, the weighted measurement fusion estimator has a large computational burden. And covariance intersection fusion avoids solving cross-covariance matrices. So it has the minimal computational burden. Lastly, A simulation example of the target tracking controllable system with two sensors shows its effectiveness and correctness.

The main structure of this paper is as follows: Problem formulation is given in Section 2. The Local optimal steady-state estimator is obtained in Section 3. In Section 4 multisensor information fusion optimal steady-state estimator is presented. A simulation example with 2-sensor is given is Section 5. In Section 6 the conclusions of this paper are given.

\section{Problem Formulation}

Consider the multi-sensor discrete time time-invariant stochastic linear system with the same observation array, the system errors and sensor errors

$$
\begin{aligned}
& \boldsymbol{x}(t+1)=\boldsymbol{\Phi} \boldsymbol{x}(t)+\boldsymbol{\Gamma} \boldsymbol{w}(t)+\boldsymbol{U d}(t) \\
& \boldsymbol{d}(t+1)=\boldsymbol{d}(t)+\boldsymbol{\zeta}(t) \\
& \boldsymbol{y}_{i}(t)=\boldsymbol{H} \boldsymbol{x}(t)+\boldsymbol{v}_{i}(t)+\boldsymbol{e}_{i}(t)
\end{aligned}
$$


$\boldsymbol{e}_{i}(t+1)=\boldsymbol{e}_{i}(t)+\boldsymbol{\xi}_{i}(t), \quad i=1, \cdots, L$

where $\boldsymbol{x}^{\boldsymbol{x}(t) \in \boldsymbol{R}^{n}}$ is the state of the system, $\boldsymbol{y}_{i}(t) \in \boldsymbol{R}^{m_{i}}$ is the measurement of the $i$ th sensor subsystem, $\boldsymbol{\Phi}, \boldsymbol{\Gamma}, \boldsymbol{U}, \boldsymbol{H}$ is the suitable dimensional matrix respectively, $\boldsymbol{d}(t) \in \boldsymbol{R}^{n_{d}}$ is system errors and $\boldsymbol{e}_{i}(t) \in \boldsymbol{R}^{m_{i}}$ is sensor errors. $\boldsymbol{\xi}_{i}(t), i=1,2, \cdots, L$ are independence white noises with zero mean, and they and $\boldsymbol{w}(t) \in \boldsymbol{R}^{r}, \boldsymbol{v}_{i}(t) \in \boldsymbol{R}^{m i}, i=1, \cdots, L$ are independence. And the subscript $i$ is the $i$ th sensor of all the sensor, $\mathrm{L}$ is the number of sensor in the system.

Assumption $1 \boldsymbol{w}(t) \in \boldsymbol{R}^{r}$ and $\boldsymbol{v}_{j}(t) \in \boldsymbol{R}^{m j}, j=1, \cdots, K$ independence white noises with zero mean and covariance are $\boldsymbol{Q}_{w}$ and $\boldsymbol{Q}_{v i}$ individually

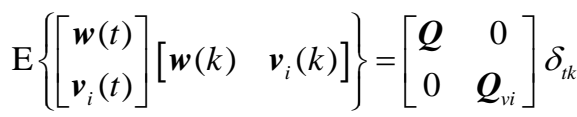

where the superscript $\mathrm{T}$ is the transpose, $\mathrm{E}$ is the expectation, $\delta_{t t}=1, \delta_{t k}=0(t \neq k)$.

Assumption $2(\boldsymbol{\Phi}, \boldsymbol{H})$ is completely observable pair, and $(\boldsymbol{\Phi}, \boldsymbol{\Gamma})$ is completely stable pair.

Assumption 3 The initial time ${ }^{t_{0}=-\infty}$.

Steady-state estimation problem is based on the measurement $\left(\boldsymbol{y}_{i}(t+N), \boldsymbol{y}_{i}(t+N-1), \cdots\right)$ , to obtain the linear minimum variance state $\hat{x}_{i}(t \mid t+N), i=1,2 \cdots L$. For $N=0, N>0$ or $N<0$, we named it as steady-state filtering, smoothing or predictor. Further weighted measurement fusion and distributed optimal information fusion steady-state estimation $\hat{x}_{0}(t \mid t+N)$ is obtained, it consists of weighted local steady-state estimators.

\section{Local Optimal Steady-State Estimator}

For the system (1) (4), we can get the following augmentation system

(6)

$$
\overline{\boldsymbol{x}}_{i}(t+1)=\overline{\boldsymbol{\Phi}}_{i} \overline{\boldsymbol{x}}_{i}(t)+\overline{\boldsymbol{\Gamma}}_{i} \overline{\boldsymbol{w}}_{i}(t)
$$

$\boldsymbol{y}_{i}(t)=\overline{\boldsymbol{H}} \bar{x}_{i}(t)+\boldsymbol{v}_{i}(t), \quad i=1, \cdots, L$

where

$$
\overline{\boldsymbol{x}}_{i}(t)=\left[\begin{array}{l}
\boldsymbol{x}(t) \\
\boldsymbol{d}(t) \\
\boldsymbol{e}_{i}(t)
\end{array}\right], \overline{\boldsymbol{w}}_{i}(t)=\left[\begin{array}{l}
\boldsymbol{w}(t) \\
\boldsymbol{\zeta}(t) \\
\boldsymbol{\xi}_{i}(t)
\end{array}\right], \overline{\boldsymbol{\Phi}}_{i}=\left[\begin{array}{ccc}
\boldsymbol{\Phi} & \boldsymbol{U} & 0 \\
0 & I_{n_{d}} & 0 \\
0 & 0 & \boldsymbol{\beta}_{i}
\end{array}\right], \overline{\boldsymbol{\Gamma}}=\left[\begin{array}{ccc}
\boldsymbol{\Gamma} & 0 & 0 \\
0 & I_{n_{d}} & 0 \\
0 & 0 & I_{m_{i}}
\end{array}\right], \overline{\boldsymbol{H}}=\left[\begin{array}{lll}
\boldsymbol{H} & 0 & I_{m}
\end{array}\right]
$$

The state $\boldsymbol{x}^{(t)}$ is the firstly $n$th components for the system (6) and (7), so, the original problem converts to the fusion steady-state estimator problem for the system (7) and (8). The system noise $\overline{\boldsymbol{w}}_{i}(t)$ and observation noise $\boldsymbol{v}_{i}(t)$ are correlated, and

$\mathrm{E}\left\{\left[\begin{array}{c}\overline{\boldsymbol{w}}_{i}(t) \\ \boldsymbol{v}_{i}(t)\end{array}\right]\left[\begin{array}{cc}\overline{\boldsymbol{w}}_{j}^{\mathrm{T}}(k) & \boldsymbol{v}_{j}^{\mathrm{T}}(k)\end{array}\right]\right\}=\left[\begin{array}{cc}\boldsymbol{Q}_{\bar{w}_{i j}} & 0 \\ 0 & \boldsymbol{Q}_{v_{i j}}\end{array}\right] \delta_{t k}$

where $Q_{\bar{w}_{i i}}=Q_{\bar{w}_{i}}=\operatorname{diag}\left(Q_{w}, Q_{\zeta}, Q_{\xi_{j}}\right), Q_{\bar{w}_{i j}}=\operatorname{diag}\left(Q_{w}, Q_{\zeta}, 0\right), i \neq j$.

From (6) and (7) having

$$
\boldsymbol{y}_{i}(t)=\overline{\boldsymbol{H}}\left(I_{n}-q^{-1} \overline{\boldsymbol{\Phi}}_{i}\right)^{-1} \overline{\boldsymbol{\Gamma}}_{i} q^{-1} \overline{\boldsymbol{w}}_{i}(t)+\boldsymbol{v}_{i}(t)
$$

where $q^{-1}$ is the lag operator for the unit, introducing left decomposition 


$$
\overline{\boldsymbol{H}}\left(I_{n}-q^{-1} \overline{\boldsymbol{\Phi}}_{i}\right)^{-1} \overline{\boldsymbol{\Gamma}}_{i} q^{-1}=\boldsymbol{A}^{(i)-1}\left(q^{-1}\right) \boldsymbol{B}^{(i)}\left(q^{-1}\right)
$$

where $\boldsymbol{A}^{(i)}\left(q^{-1}\right)$ and $\boldsymbol{B}^{(i)}\left(q^{-1}\right)$ is matrix polynomial, it is shaped as $X^{i)}\left(q^{-1}\right)=x_{0}^{(i)}+x_{1}^{(i)} q^{-1}+$ $\cdots+x_{n_{i j}}^{(i)} q^{-n_{n_{i}}}$, and $A_{0}^{(i)}=I_{m i}, B_{0}^{(i)}=0$, letting $\boldsymbol{X}^{(i)}-1\left(q^{-1}\right)=\left(\boldsymbol{X}^{(i)}\left(q^{-1}\right)\right)^{-1}$

Substituting (11) into (10) yields

$\boldsymbol{A}^{(i)-1}\left(q^{-1}\right) \boldsymbol{y}_{i}(t)=\boldsymbol{B}^{(j)}\left(q^{-1}\right) \overline{\boldsymbol{w}}_{i}(t)+\boldsymbol{A}^{(i)-1}\left(q^{-1}\right) \boldsymbol{v}_{i}(t)$

For $\left(\boldsymbol{A}^{(i)}\left(q^{-1}\right), \boldsymbol{B}^{(i)}\left(q^{-1}\right)\right)$ is left and Assumption 1, yielding the ARMA innovation model

$\boldsymbol{A}^{(i)-1}\left(q^{-1}\right) \boldsymbol{y}_{i}(t)=\boldsymbol{D}^{(i)}\left(q^{-1}\right) \boldsymbol{\varepsilon}_{i}(t)$

where innovation $\varepsilon_{i}(t) \in R^{m i}$ is white noises with zero mean, and its covariance is $Q_{\varepsilon i}$. $\boldsymbol{D}^{(i)}\left(q^{-1}\right)$ is stable, $\boldsymbol{D}_{0}^{(i)}=I_{m}$, and having

$\boldsymbol{D}^{(i)}\left(q^{-1}\right) \boldsymbol{\varepsilon}_{i}(t)=\boldsymbol{B}^{(i)}\left(q^{-1}\right) \overline{\boldsymbol{w}}_{i}(t)+\boldsymbol{A}^{(i)-1}\left(q^{-1}\right) \boldsymbol{v}_{i}(t)$

$\boldsymbol{D}^{(i)}\left(q^{-1}\right)$ and $Q_{\varepsilon i}$ are computed through G-W algorithm.

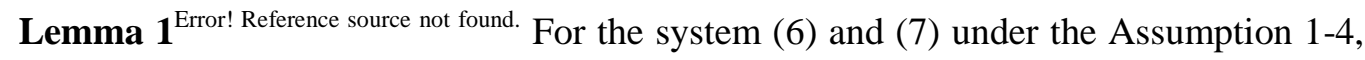
the $i$ th sensor subsystem has the local optimal steady-state Kalman filtering equations:

$\hat{\boldsymbol{x}}_{i}(t+1 \mid t+1)=\boldsymbol{\Psi}_{f i} \hat{\boldsymbol{x}}_{i}(t \mid t)+\left[\boldsymbol{I}_{n}-\boldsymbol{K}_{f i} \overline{\boldsymbol{H}}\right] \boldsymbol{y}_{i}(t)+\boldsymbol{K}_{f i} \boldsymbol{y}_{i}(t+1)$

$\boldsymbol{\Psi}_{f i}=\left[\boldsymbol{I}_{n}-\boldsymbol{K}_{f i} \overline{\boldsymbol{H}}\right] \overline{\boldsymbol{\Phi}}_{i}$

where $\boldsymbol{\Psi}_{f i}$ is a stable matrix, the filtering gain $\boldsymbol{K}_{f i}$ is as follows:

$\boldsymbol{K}_{f i}=\left[\begin{array}{c}\overline{\boldsymbol{H}} \\ \overline{\boldsymbol{H}} \overline{\boldsymbol{\Phi}} \\ \vdots \\ \overline{\boldsymbol{H}} \overline{\boldsymbol{\Phi}}^{\beta_{i}-1}\end{array}\right]^{+}\left[\begin{array}{c}\boldsymbol{I}_{m_{1}}-\boldsymbol{Q}_{v i} \\ \boldsymbol{M}_{1}^{(i)} \\ \vdots \\ \boldsymbol{M}_{\beta_{i}-1}^{(i)}\end{array}\right]$

The pseudo inverse $\boldsymbol{X}^{+}$of the matrix $\boldsymbol{X}$ is defined as $\boldsymbol{X}^{+}=\left(\boldsymbol{X}^{\mathrm{T}} \boldsymbol{X}\right)^{-1} \boldsymbol{X}^{\mathrm{T}}, \beta_{i}$ is index of correlation of $(\overline{\boldsymbol{\Phi}}, \overline{\boldsymbol{H}})$, the coefficient matrix ${ }^{(i)}$ can be recursively computed as

$\boldsymbol{M}_{k}^{(i)}=-\boldsymbol{A}_{i 1} \boldsymbol{M}_{k-1}^{(i)}-\cdots-\boldsymbol{A}_{i n_{i i}} \boldsymbol{M}_{k-n_{a i}}^{(i)}+\boldsymbol{D}_{i k}$

where $\boldsymbol{M}_{0}^{(i)}=I_{m_{i}}, \boldsymbol{M}_{k}^{(i)}=0(k<0), \boldsymbol{D}_{i k}=0\left(k>n_{d i}\right)$.

Steady local filtering error covariance matrix $\boldsymbol{P}_{i}$ satisfies the Lyapunov equation

$\boldsymbol{P}_{i}=\boldsymbol{\Psi}_{f i} \boldsymbol{P}_{i j} \boldsymbol{\Psi}_{f i}^{\mathrm{T}}+\left[\boldsymbol{I}_{n}-\boldsymbol{K}_{f i} \overline{\boldsymbol{H}}\right] \overline{\boldsymbol{\Gamma}} \boldsymbol{Q}_{\bar{w}_{i i}} \overline{\boldsymbol{\Gamma}}^{\mathrm{T}}\left[\boldsymbol{I}_{n}-\boldsymbol{K}_{f i} \overline{\boldsymbol{H}}\right]^{\mathrm{T}}+\boldsymbol{K}_{f i} \boldsymbol{Q}_{v i} \boldsymbol{K}_{f i}^{\mathrm{T}}$

the cross covariance $\boldsymbol{P}_{i j}$ between any two local filtering satisfies Lyapunov equation:

$\boldsymbol{P}_{i j}=\boldsymbol{\Psi}_{f i} \boldsymbol{P}_{i j} \boldsymbol{\Psi}_{f j}^{\mathrm{T}}+\left[\boldsymbol{I}_{n}-\boldsymbol{K}_{f i} \overline{\boldsymbol{H}}\right] \overline{\boldsymbol{\Gamma}} \boldsymbol{Q}_{\bar{w}_{i i}} \overline{\boldsymbol{\Gamma}}^{\mathrm{T}}\left[\boldsymbol{I}_{n}-\boldsymbol{K}_{f j} \overline{\boldsymbol{H}}\right]^{\mathrm{T}}$

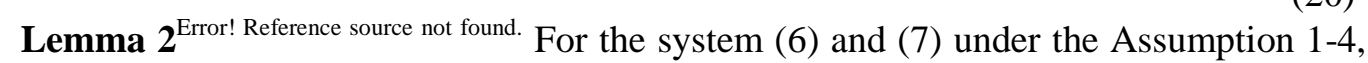
the $i$ th sensor subsystem has the local optimal steady-state Kalman prediction equations:

$\hat{\boldsymbol{x}}_{i}(t+1 \mid t)=\boldsymbol{\Psi}_{p i} \hat{\boldsymbol{x}}_{i}(t \mid t-1)+\boldsymbol{K}_{p i} \boldsymbol{y}_{i}(t)$

$\boldsymbol{\Psi}_{p i}=\overline{\boldsymbol{\Phi}}_{i}-\boldsymbol{K}_{p i} \overline{\boldsymbol{H}}$ 
where $\boldsymbol{\Psi}_{p i}$ is a stable matrix, the prediction gain $\boldsymbol{K}_{p i}$ is as follows:

$\boldsymbol{K}_{p i}=\left[\begin{array}{c}\overline{\boldsymbol{H}} \\ \overline{\boldsymbol{H}} \overline{\boldsymbol{\Phi}} \\ \vdots \\ \overline{\boldsymbol{H}} \overline{\boldsymbol{\Phi}}^{\beta_{i}-1}\end{array}\right]^{+}\left[\begin{array}{c}\boldsymbol{M}_{1}^{(i)} \\ \boldsymbol{M}_{2}^{(i)} \\ \vdots \\ \boldsymbol{M}_{\beta_{i}}^{(i)}\end{array}\right]$

where the coefficient matrix $\boldsymbol{M}_{k}^{(i)}$ can be recursively computed by (18).

Steady local prediction error covariance matrix ${ }^{\Sigma_{i}}$ satisfies the Lyapunov equation

$\boldsymbol{\Sigma}_{i}=\boldsymbol{\Psi}_{p i} \boldsymbol{\Sigma}_{i} \boldsymbol{\Psi}_{p i}^{\mathrm{T}}+\overline{\boldsymbol{\Gamma}} \boldsymbol{Q}_{\bar{w}_{i i}} \overline{\boldsymbol{\Gamma}}^{\mathrm{T}}+\boldsymbol{K}_{p i} \boldsymbol{Q}_{v i} \boldsymbol{K}_{p i}^{\mathrm{T}}$

the cross covariance ${ }^{\Sigma_{i j}}$ between any two local prediction satisfies Lyapunov equation:

$\boldsymbol{\Sigma}_{i j}=\boldsymbol{\Psi}_{p i} \boldsymbol{\Sigma}_{i j} \boldsymbol{\Psi}_{p j}^{\mathrm{T}}+\overline{\boldsymbol{\Gamma}} \boldsymbol{Q}_{\bar{w}_{i i}} \overline{\boldsymbol{\Gamma}}^{\mathrm{T}}$

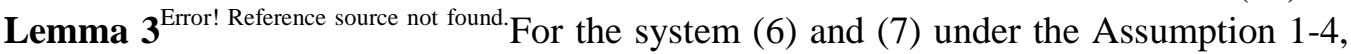
the $i$ th sensor subsystem has the local $(-N)$-step-ahead optimal steady-state Kalman prediction equations:

$\hat{\boldsymbol{x}}_{i}(t \mid t+N)=\overline{\boldsymbol{\Phi}}^{-N-1} \hat{\boldsymbol{x}}_{i}(t+N+1 \mid t+N), N \leq 2, \quad i=1, \cdots, L$

Steady local filter error covariance matrix $\boldsymbol{P}_{i}(N)$ can be calculated by

$\boldsymbol{P}_{i}(N)=\overline{\boldsymbol{\Phi}}^{-N-1} \boldsymbol{\Sigma}_{i} \overline{\boldsymbol{\Phi}}^{(-N-1) \mathrm{T}}+\sum_{j=0}^{-N-2} \overline{\boldsymbol{\Phi}}^{j} \boldsymbol{\Gamma} \boldsymbol{Q} \boldsymbol{\Gamma}^{\mathrm{T}} \overline{\boldsymbol{\Phi}}^{j \mathrm{~T}}, N \leq 2$

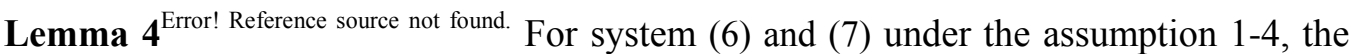
$i$ th sensor subsystem has the local optimal steady-state Kalman smoothing equations:

$\hat{\boldsymbol{x}}_{i}(t \mid t+N)=\hat{\boldsymbol{x}}_{i}(t \mid t-1)+\sum_{k=0}^{N} \boldsymbol{K}_{m i}(k) \boldsymbol{\varepsilon}_{i}(t+k) \quad, i=1, \cdots, L$

where $\hat{\boldsymbol{x}}_{i}(t \mid t-1)$ and $\boldsymbol{\varepsilon}_{i}(t)=\boldsymbol{y}_{i}(t)-\overline{\boldsymbol{H}} \hat{\boldsymbol{x}}_{i}(t \mid t-1)$ can be computed by Lemma 2.

The smoothing gain $\boldsymbol{K}_{m i}$ is as follows:

$\boldsymbol{K}_{m i}(k)=\boldsymbol{\Sigma}_{i} \boldsymbol{\Psi}_{p i}^{\mathrm{Tk}} \overline{\boldsymbol{H}}^{\mathrm{T}} \boldsymbol{Q}_{\varepsilon i}^{-1}$

$\boldsymbol{Q}_{\varepsilon i}=\overline{\boldsymbol{H}} \Sigma_{i} \overline{\boldsymbol{H}}^{\mathrm{T}}+\boldsymbol{Q}_{v i}$

$\boldsymbol{K}_{p i}=\overline{\boldsymbol{\Phi}} \Sigma_{i} \overline{\boldsymbol{H}}_{i}^{\mathrm{T}} \boldsymbol{Q}_{\varepsilon i}^{-1}$

$\Sigma_{i}$ can be computed by Lemma 2 .

Steady local smoothing error covariance matrix $\boldsymbol{P}_{i}(N)$ satisfies the Lyapunov equation $\boldsymbol{P}_{i}(N)=\boldsymbol{\Sigma}_{i}-\sum_{k=0}^{N} \boldsymbol{K}_{m i}(k) \boldsymbol{Q}_{\varepsilon i} \boldsymbol{K}_{m i}^{\mathrm{T}}(k)$

the cross covariance $\boldsymbol{P}_{i j}(N)$ between any two local filtering satisfies Lyapunov equation: 


$$
\boldsymbol{P}_{i j}(N)=\boldsymbol{\Sigma}_{i j}-\sum_{r=0}^{N} \boldsymbol{K}_{m i}(r) \overline{\boldsymbol{H}} \boldsymbol{\Psi}_{p i}^{r} \boldsymbol{\Sigma}_{i j}-\sum_{s=0}^{N} \boldsymbol{\Sigma}_{i j} \boldsymbol{\Psi}_{p j}^{\mathrm{T} s} \overline{\boldsymbol{H}} \boldsymbol{K}_{m j}^{\mathrm{T}}(s) \overline{\boldsymbol{H}} \boldsymbol{\Psi}_{p i}^{r} \boldsymbol{\Sigma}_{i j}+\sum_{r=0}^{N} \sum_{s=0}^{N} \boldsymbol{K}_{m i}(r) \boldsymbol{E}_{i j}(r, s) \boldsymbol{K}_{m j}^{\mathrm{T}}(s)
$$

where ${ }^{\Sigma_{i j}}$ can be calculated by (25).

$$
\begin{aligned}
& \boldsymbol{E}_{i j}(r, s)=\overline{\boldsymbol{H}} \boldsymbol{\Psi}_{p i}^{r} \boldsymbol{\Sigma}_{i j} \boldsymbol{\Psi}_{p j}^{\mathrm{Ts}} \overline{\boldsymbol{H}}^{\mathrm{T}}+\sum_{k=1}^{\min (r, s)} \overline{\boldsymbol{H}} \boldsymbol{\Psi}_{p i}^{r-k}\left[\overline{\boldsymbol{\Gamma}},-\boldsymbol{K}_{p i}\right]\left[\begin{array}{cc}
\boldsymbol{Q}_{\bar{w}_{i j}} & 0 \\
0 & \overline{\boldsymbol{Q}}_{v_{i j}}
\end{array}\right]\left[\begin{array}{c}
\overline{\boldsymbol{\Gamma}} \\
-\boldsymbol{K}_{p i}
\end{array}\right]^{\mathrm{T}} \boldsymbol{\Psi}_{p j}^{\mathrm{T}(s-k)} \overline{\boldsymbol{H}}^{\mathrm{T}} \\
& i \neq j, \min (r, s)>0 \\
& \quad \text { If } \min (r, s)=0 \text {, then having } \\
& \boldsymbol{E}_{i j}(0,0)=\overline{\boldsymbol{H}} \boldsymbol{\Sigma}_{i j} \overline{\boldsymbol{H}}^{\mathrm{T}} \\
& \boldsymbol{E}_{i j}(r, 0)=\overline{\boldsymbol{H}} \boldsymbol{\Psi}_{p i}^{r} \boldsymbol{\Sigma}_{i j} \overline{\boldsymbol{H}}^{\mathrm{T}} \\
& \boldsymbol{E}_{i j}(0, s)=\overline{\boldsymbol{H}} \boldsymbol{\Sigma}_{i j} \boldsymbol{\Psi}_{p j}^{\mathrm{Ts}} \overline{\boldsymbol{H}}^{\mathrm{T}}
\end{aligned}
$$

\section{Multi-Sensor Information Fusion Optimal Steady-State Estimator}

The system (7) can be written as

$$
\begin{aligned}
& \overline{\boldsymbol{y}}^{(\mathrm{o})}(t)=\overline{\boldsymbol{H}}^{(\mathrm{o})} \boldsymbol{x}(t)+\overline{\boldsymbol{v}}^{(\mathrm{o})}(t) \\
& \overline{\boldsymbol{y}}^{(\mathrm{o})}(t)=\left[\sum_{i=1}^{L} \boldsymbol{Q}_{v i}^{-1}\right]^{-1} \sum_{i=1}^{L} \boldsymbol{Q}_{v i}^{-1} \boldsymbol{y}_{i}(t) \\
& \overline{\boldsymbol{v}}^{(\mathrm{o})}(t)=\left[\sum_{i=1}^{L} \boldsymbol{Q}_{v i}^{-1}\right]^{-1} \sum_{i=1}^{L} \boldsymbol{Q}_{v i}^{-1} \boldsymbol{v}_{i}(t) \\
& \boldsymbol{Q}_{v}^{(\mathrm{o})}=\left[\sum_{i=1}^{L} \boldsymbol{Q}_{v i}^{-1}\right]^{-1}
\end{aligned}
$$

Theorem 1 For the system (6) and (38) under the Assumption 1-4, global optimality weighted measurement fusion steady-state Kalman filtering equations:

$$
\begin{aligned}
& \hat{\boldsymbol{x}}^{(\mathrm{o})}(t+1 \mid t+1)=\boldsymbol{\Psi}_{f} \hat{\boldsymbol{x}}^{(\mathrm{o})}(t \mid t)+\boldsymbol{K}_{f} \overline{\boldsymbol{y}}^{(\mathrm{o})}(t) \\
& \boldsymbol{\Psi}_{f}=\left[\boldsymbol{I}_{n}-\boldsymbol{K}_{f} \overline{\boldsymbol{H}}^{(\mathrm{o})}\right] \overline{\boldsymbol{\Phi}}
\end{aligned}
$$

Where $\boldsymbol{\Psi}_{f i}$ a is a stable matrix, the filtering gain $\boldsymbol{K}_{f i}$ is as follows:

$$
\boldsymbol{K}_{f i}=\left[\begin{array}{c}
\overline{\boldsymbol{H}}^{(\mathrm{o})} \\
\overline{\boldsymbol{H}}^{(\mathrm{o})} \overline{\boldsymbol{\Phi}} \\
\vdots \\
\overline{\boldsymbol{H}}^{(\mathrm{o})} \overline{\boldsymbol{\Phi}}^{\beta-1}
\end{array}\right]^{+}\left[\begin{array}{c}
I_{m_{1}}-\boldsymbol{Q}_{v} \\
\boldsymbol{M}_{1} \\
\vdots \\
\boldsymbol{M}_{\beta_{i}-1}
\end{array}\right]
$$

The pseudo inverse $X^{+}$of the matrix $X$ is defined as $X^{+}=\left(X^{\mathrm{T}} X\right)^{-1} X^{\mathrm{T}}, \beta$ is index of correlation of $\left(\overline{\boldsymbol{\Phi}}, \overline{\boldsymbol{H}}^{(\mathrm{o})}\right)$, the coefficient matrix $\boldsymbol{M}_{k}$ can be recursively computed as

$$
\boldsymbol{M}_{k}=-\boldsymbol{A}_{1} \boldsymbol{M}_{k-1}-\cdots-\boldsymbol{A}_{n_{a}} \boldsymbol{M}_{k-n_{a}}+\boldsymbol{D}_{k}
$$

where $\boldsymbol{M}_{0}=I_{m_{i}}, \boldsymbol{M}_{k}=0(k<0), \quad \boldsymbol{D}_{k}=0\left(k>n_{d}\right)$.

Optimality weighted measurement fusion filtering error covariance matrix $\boldsymbol{P}_{0}$ satisfies the Lyapunov equation 
$\boldsymbol{P}_{0}=\boldsymbol{\Psi}_{f} \boldsymbol{P}_{0} \boldsymbol{\Psi}_{f}^{\mathrm{T}}+\left[\boldsymbol{I}_{n}-\boldsymbol{K}_{f} \overline{\boldsymbol{H}}^{(\mathrm{o})}\right] \overline{\boldsymbol{\Gamma}} \boldsymbol{Q}_{\bar{w}} \overline{\boldsymbol{\Gamma}}^{\mathrm{T}}\left[\boldsymbol{I}_{n}-\boldsymbol{K}_{f} \overline{\boldsymbol{H}}^{(\mathrm{o})}\right]^{\mathrm{T}}+\boldsymbol{K}_{f} \boldsymbol{Q}_{v}^{(\mathrm{o})} \boldsymbol{K}_{f}^{\mathrm{T}}$

And global optimality weighted measurement fusion steady-state Kalman prediction equations:

$\hat{\boldsymbol{x}}^{(\mathrm{o})}(t+1 \mid t)=\boldsymbol{\Psi}_{p} \hat{\boldsymbol{x}}^{(\mathrm{o})}(t \mid t-1)+\boldsymbol{K}_{p} \boldsymbol{y}(t)$

$\boldsymbol{\Psi}_{p}=\overline{\boldsymbol{\Phi}}-\boldsymbol{K}_{p} \overline{\boldsymbol{H}}^{(\mathrm{o})}$

where $\boldsymbol{\Psi}_{p}$ is a stable matrix, the prediction gain $\boldsymbol{K}_{p}$ is as follows:

$\boldsymbol{K}_{p}=\left[\begin{array}{c}\overline{\boldsymbol{H}}^{(\mathrm{o})} \\ \overline{\boldsymbol{H}}^{(\mathrm{o})} \overline{\boldsymbol{\Phi}} \\ \vdots \\ \overline{\boldsymbol{H}}^{(\mathrm{o})} \overline{\boldsymbol{\Phi}}^{\beta-1}\end{array}\right]^{+}\left[\begin{array}{c}\boldsymbol{M}_{1} \\ \boldsymbol{M}_{2} \\ \vdots \\ \boldsymbol{M}_{\beta}\end{array}\right]$

where the coefficient matrix $\boldsymbol{M}_{k}$ can be recursively computed by (45)

Optimality weighted measurement fusion prediction error covariance matrix $\Sigma_{0}$ satisfies the Lyapunov equation

$\boldsymbol{\Sigma}_{0}=\boldsymbol{\Psi}_{p} \boldsymbol{\Sigma}_{0} \boldsymbol{\Psi}_{p}^{\mathrm{T}}+\overline{\boldsymbol{\Gamma}} \boldsymbol{Q}_{\bar{w}} \overline{\boldsymbol{\Gamma}}^{\mathrm{T}}+\boldsymbol{K}_{p} \boldsymbol{Q}_{v}^{(\mathrm{o})} \boldsymbol{K}_{p}^{\mathrm{T}}$

And global optimality weighted measurement fusion steady-state Kalman smoothing equations:

$\hat{\boldsymbol{x}}^{(\mathrm{o})}(t \mid t+N)=\hat{\boldsymbol{x}}^{(\mathrm{o})}(t \mid t-1)+\sum_{k=0}^{N} \boldsymbol{K}_{m}(k) \boldsymbol{\varepsilon}(t+k)$

$\boldsymbol{K}_{m}(k)=\boldsymbol{\Sigma}_{0} \boldsymbol{\Psi}_{p}^{\mathrm{Tk}} \overline{\boldsymbol{H}}^{(\mathrm{o}) \mathrm{T}} \boldsymbol{Q}_{\varepsilon}^{-1}$

$\boldsymbol{Q}_{\varepsilon}=\overline{\boldsymbol{H}}^{(\mathrm{o})} \boldsymbol{\Sigma}_{0} \overline{\boldsymbol{H}}^{(\mathrm{o}) \mathrm{T}}+\boldsymbol{Q}_{v}^{(\mathrm{o})}$

$\boldsymbol{\Psi}_{p}=\overline{\boldsymbol{\Phi}}-\boldsymbol{K}_{p} \overline{\boldsymbol{H}}^{(\mathrm{o})}$

where $\boldsymbol{K}_{p}$ can be calculated by (49).

Optimality weighted measurement fusion prediction error covariance matrix $\boldsymbol{P}_{i}(N)$ satisfies the Lyapunov equation

$\boldsymbol{P}_{0}(N)=\boldsymbol{\Sigma}_{0}-\sum_{k=0}^{N} \boldsymbol{K}_{m}(k) \boldsymbol{Q}_{\varepsilon} \boldsymbol{K}_{m}^{\mathrm{T}}(k)$

Theorem 2 For the system (6) and (7), under the Assumption $1-4$, the optimal fused steady-state Kalman filtering $\hat{\boldsymbol{x}}_{0}(t \mid t)$ weighted by scalars is given
as

$\hat{\boldsymbol{x}}_{0}(t \mid t)=\sum_{i=1}^{l} \alpha_{i} \hat{\boldsymbol{x}}_{i}(t \mid t)$

Under the linear minimum variance optimal information fusion criterion which minimize the performance index, the optimal weighting coefficients $\alpha_{i}, i=1,2, \cdots, L$ are given by 


$$
\left[\alpha_{1}, \cdots, \alpha_{l}\right]=\frac{\boldsymbol{e}^{\mathrm{T}} \boldsymbol{P}_{\mathrm{tr}}^{-1}}{\boldsymbol{e}^{\mathrm{T}} \boldsymbol{P}_{\mathrm{tr}}^{-1} \boldsymbol{e}}
$$

where we define the $L \times L$ matrix $\boldsymbol{P}_{\mathrm{tr}}=\left(\operatorname{tr} \boldsymbol{P}_{i j}\right)_{L \times L}, i, j=1,2, \cdots, L$, and $\boldsymbol{P}_{i j}$ can be calculated by (20), and $L \times 1$ row vector $\boldsymbol{e}=\left[\begin{array}{lll}1 & \cdots & 1\end{array}\right]^{\mathrm{T}}$.

The optimal fused variance matrix is given as

$$
\boldsymbol{P}_{0}(N)=\sum_{i, j=1}^{L} \alpha_{i} \alpha_{j} \boldsymbol{P}_{i j}(N)
$$

and

$\operatorname{tr} \boldsymbol{P}_{0} \leq \operatorname{tr} \boldsymbol{P}_{i}, \quad i=1,2, \cdots, L$

Theorem 3 For the system (6) and (7), under the same conditions, when the variance of $\mathrm{P}_{1}$ and $\mathrm{P}_{2}$ are known, but the cross covariance $\mathrm{P}_{12}$ is unknown, using the covariance intersection $(\mathrm{CI})$ fusion method, this paper proposes a suboptimal fusion Kalman estimators is as follows:

$\hat{\boldsymbol{x}}_{C I}(t \mid t+N)=\boldsymbol{P}_{C I}\left[\omega \boldsymbol{P}_{1}^{-1} \hat{\boldsymbol{x}}_{1}(t \mid t+N)+(1-\omega) \boldsymbol{P}_{2}^{-1} \hat{\boldsymbol{x}}_{2}(t \mid t+N)\right]$

where ${ }^{\omega \in[0,1]}$ and minimizes the performance index

$J=\min _{\omega} \operatorname{tr} \boldsymbol{P}_{C I}$

and $\boldsymbol{P}_{C I}$ is defined as

$\boldsymbol{P}_{C I}=\left[\omega \boldsymbol{P}_{1}^{-1}+(1-\omega) \boldsymbol{P}_{2}^{-1}\right]^{-1}$

For the non-linear optimization problems (62), the optimal weights $\omega$ can be obtained by 0.618 method or the Fabonacci method.

\section{Simulation Example}

Consider 2-sensor discrete-time linear time-invariant stochastic tracking system (6) and (7), where $T=0.7$ is the sampled period,

$$
A=\left[\begin{array}{ll}
1 & \mathrm{~T} \\
0 & 1
\end{array}\right], \quad \boldsymbol{\Gamma}=\left[\begin{array}{ll}
\mathrm{T}^{2} / 2 & \mathrm{~T}
\end{array}\right]^{\mathrm{T}}, \quad H=\left[\begin{array}{ll}
1 & 0
\end{array}\right] \text {. And }
$$

$w(t)$ is white noises with zero mean and its covariance is $\sigma_{w}^{2}=0.1 . v_{i}(t)$ and $w(t)$ are independence noise, $\sigma_{v 1}^{2}=0.1, \sigma_{v 2}^{2}=0.5$.

Steady-state estimation problem is based on the measurement $\left(\boldsymbol{y}_{i}(t+N), \boldsymbol{y}_{i}(t+N-1), \cdots\right)$ , to obtain the linear minimum variance state $\hat{x}_{i}(t \mid t+N)$ and optimal information fusion steady-state estimation $\hat{x}_{0}(t \mid t+N)$. 


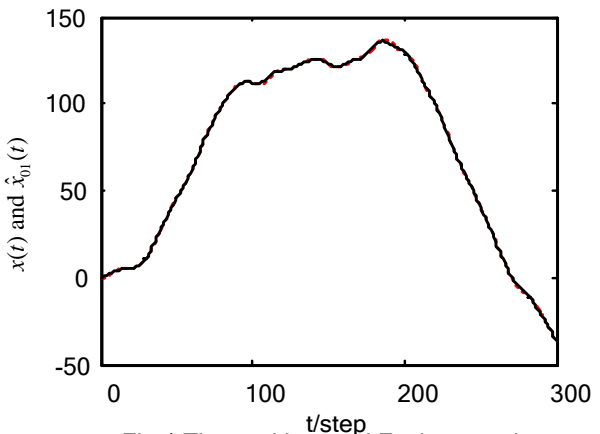

Fig. 1 The position and Fusion steadystate weighted measurement fusion filter

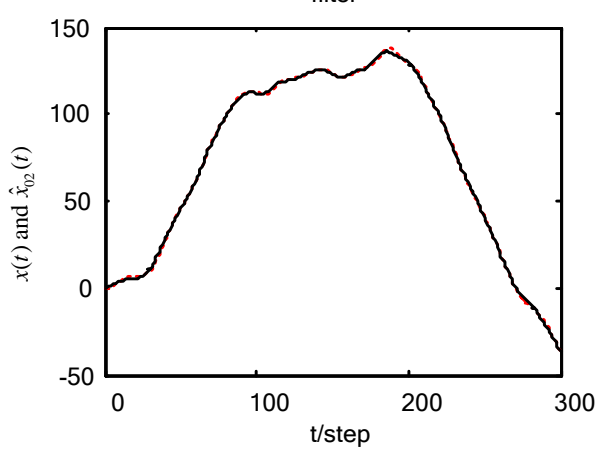

Fig. 3 The position and Fusion steadystate fusion filter weighted by scalar

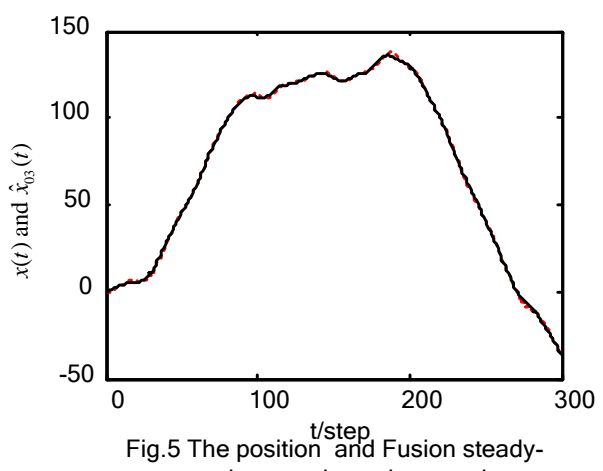

state the covariance intersection fusion filter

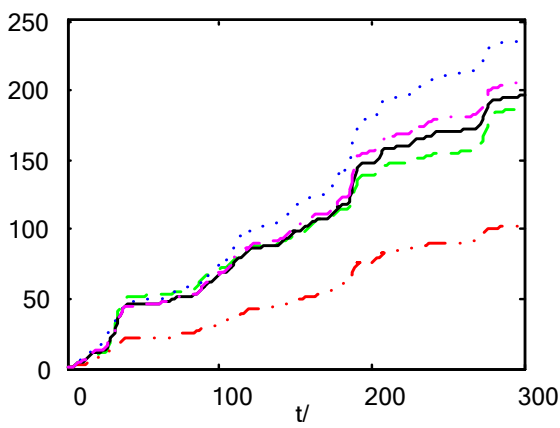

Fig.7 The curves oftere sum of absolute error curve for local and fusion filters of the
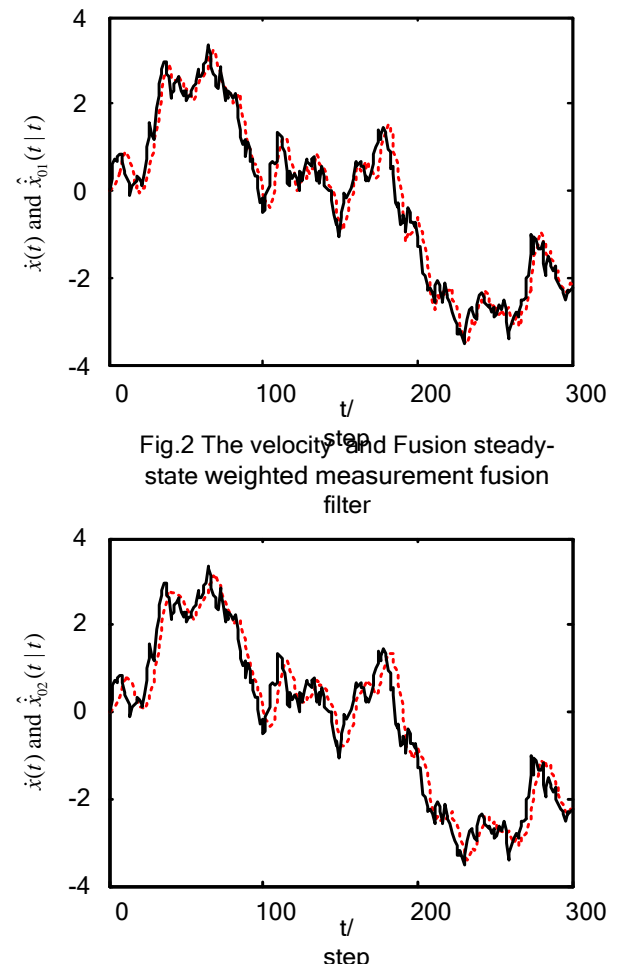

Fig.4 The velocity and Fusion steadystate fusion filter weighted by scalar

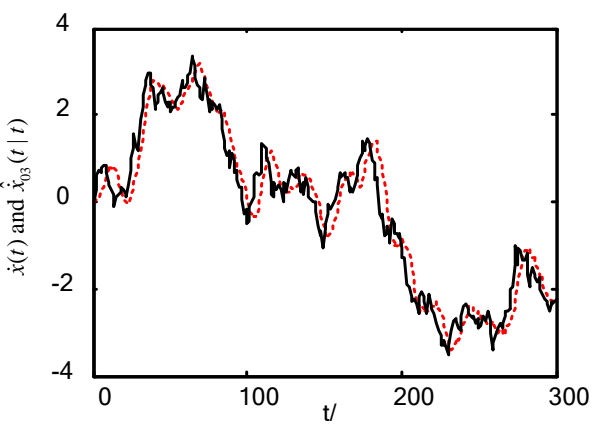

Fig.6 The velocitstegrad Fusion steadystate the covariance intersection fusion filter

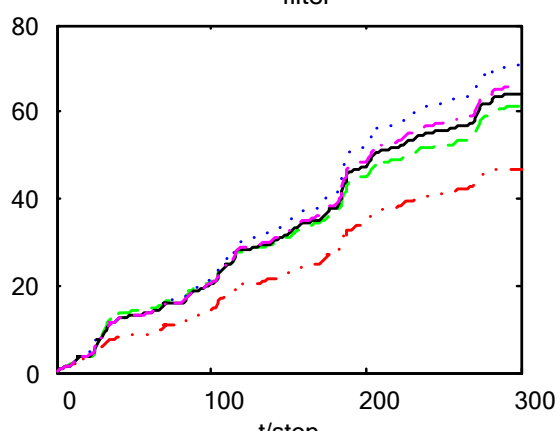

Fig.8 The curves of the sum of absolute error curve for local and fusion filters of the

$$
\begin{aligned}
& \text { position } \\
& \text { weıgntea measurement }{ }^{\text {velocity }}
\end{aligned}
$$

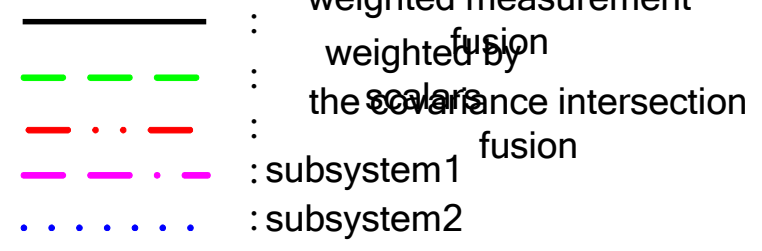


The simulation results are shown in Figure 1-Figure 8. Figure 1 to 6 gives the fusion filter weighted measurement fusion, weighted by scalars and the covariance intersection fusion. Figure 7 and Figure 8 are the absolute error curve for the steady-state filter and fusion steady-state filter of position and velocity weighted measurement fusion, weighted by scalars and the covariance. In the figure, the accuracy of the fusion state filter is higher than any of the single sensor. The accuracy of above three kinds of weighted fusion estimator from high to low is the weighted measurement fusion, weighted by scalars, and covariance intersection fusion. But the computational burden is on the contrary, the weighted measurement fusion estimator has a large computational burden. And covariance intersection fusion avoids solving cross-covariance matrices and has the minimal computational burden.

\section{Conclusions}

In this paper, a multi-sensor information fusion steady-state Kalman estimator for discrete time stochastic linear systems with system errors and sensor errors is presented. Information fusion rule, which adopted in this paper, includes weighted measurement fusion, weighted by scalars and the covariance intersection fusion. The estimation accuracy for the system is greatly improved compared with the single local sensor. Weighted measurement fusion, scalars weighted and the covariance intersection fusion, the accuracy of above three kinds of weighted fusion filtering from high to low. But the computational burden is on the contrary. Fusion filtering weighted by weighted measurement fusion has a large computational burden, and the covariance intersection fusion with minimal computational burden, and it is suitable for real-time applications. The simulation example shows its validity. The algorithm presented in this paper has many advantages.

\section{Acknowledgment}

This work is supported by the National Natural Science Foundation of China Youth Fund Project under Grant NSFC-61503127, by the Natural Science Foundation of Heilongjiang Province of China (Grant Nos. F2015014).

*Corresponding author: Gang Hao.

\section{References}

[1] Sun Shuli, Cui Pingyuan, Multi-sensor optimal information fusion steady-state Kalman filter weighted by scalars, CONTROL AND DECISION, 34, 3(2008) . pp. 208-211.

[2] Deng Zili, Gu Lei, Ran Chenjian, Measurement Fusion Steady-State Kalman Filtering Algorithm with Correlated Noises and Global Optimdity, 31, 3(2009) . pp. 556-560.

[3] RAN Chenjian, HUI Yusong, GU Lei, DENG ZiLi, Correlated Measurement Fusion Steady-state Kalman Filtering Algorithms and Their Optimality, automatic, 34, 3(2008) . pp. 233-239.

[4] Zhang Peng, Qi Wenjuan, Deng Zili, Gao Yuan, Liu Jinfang, Covariance intersection fusion robust steady-state Kalman filter, Control and Decision, 27, 6(2012) . pp. 904-908.

[5] P. J. Dua, S. C. Liu, J. S. Xia, Y. D. Zhao. Information fusion techniques for change detection from multi-temporal remote sensing images. Information Fusion, 14, 1 (2013). pp. 19-27.

[6] B. Khaleghi, A. Khamis, F. O. Karray, S. N. Razavi. Multisensor data fusion: A review of the state-ofthe-art. Information Fusion, 14, 1 (2013). pp. 28-44.

[7] P. Cui, H. S. Zhang, H. X. Wang, J. E. Feng. Globally optimal real-time distributed fusion of multichannel observation systems. Control Theory \& Applications, 8, 6, (2014).pp.384 - 388.

[8] M. B. Trawicki, M. T. Johnson. Distributed multichannel speech enhancement with minimum meansquare error short-time spectral amplitude, log-spectral amplitude, and spectral phase estimation. Signal Processing, 92, 2 (2012). pp. 345-356.

[9] Qi Wenjuan, Zhang Peng, Deng Zili, Robust Sequential Covariance Intersection Fusion Kalman Filtering over Multi-agent Sensor Networks with Measurement Delays and Uncertain Noise Variances, 40, 11(2014) . pp. 2632-2642.

[10] Z. L. Deng, P. Zhang, W. J. Qi, Y. Gao, J. F. Liu. The accuracy comparison of multisensor covariance intersection fuser and three weighting fusers. Information Sciences, 14, 2 (2013). pp. 177-185. 
[11] W. J. Qi, P. Zhang, Z. L. Deng. Two-level robust sequential covariance intersection fusion Kalman predictors over clustering sensor networks with uncertain noise variances. International Journal of Sensor Networks, 14, 4 (2013). pp. 251-261.

[12] W. J. Qi, P. Zhang, Z. L. Deng. Covariance Intersection Fusion Kalman Estimators for Multi-Sensor System with Colored Measurement Noises. Research Journal of Applied Sciences, Engineering and Technology 6(10): 2013. pp. 1872-1878.

[13] Deng ZiLi, Information Fusion Estimation Theory and its Application, Harbin, Science Press, (2012): $25-65$

[14] Sun ShuLi, Deng Zili. Multi-sensor Optimal Information Fusion Kalman Filter, Automatica, (2004), vol. 40, pp. 1017-1023. 


\section{Authors}

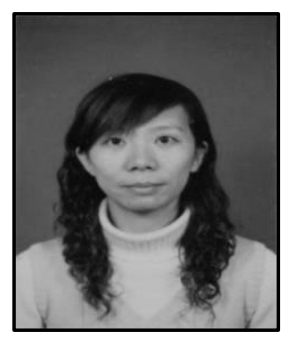

Yun $\mathbf{L i}$, She is an associate professor at Harbin University of Commerce now. She obtained her bachelor's degree and master's degree in Heilongjiang University. Her major researches are state estimation, information fusion, etc.

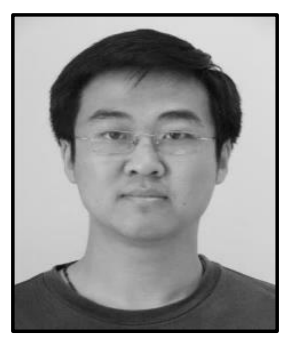

Gang Hao, $\mathrm{He}$ is an associate professor at Heilongjiang University now. He obtained his bachelor's degree and master's degree in Heilongjiang University, and obtained his Ph.D. in Harbin Engineering University. His major researches are state estimation, information fusion, etc.

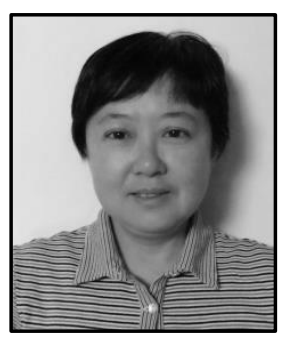

Ming Zhao, She is associate professor at Harbin University of Commerce now. She obtained her bachelor's degree and master's degree in Harbin Engineering University. Her major researches are pattern recognition, information fusion, etc. 
International Journal of Security and Its Applications

Vol. 10, No. 2 (2016) 